\title{
MASTITIS
}

https://doi.org/10.29289/259453942020V30S1093

\section{IDIOPATHIC GRANULOMATOUS MASTITIS: LITERATURE REVIEW AND RETROSPECTIVE ANALYSIS OF THE SAMPLE AT HOSPITAL FEDERAL DA LAGOA FROM 2013 TO 2017}

Michele Catucá Medeiros', Rafael Henrique Szymanski Machado

${ }^{1}$ Hospital Federal da Lagoa - Rio de Janeiro (RJ), Brazil.

Introduction: Idiopathic Granulomatous Mastitis (IGM) is a benign inflammatory, rare and chronic condition. It constitutes about $24 \%$ of all breast inflammatory diseases, and its recurrence ranges from $16 \%$ to $50 \%$. Its etiology was not completely elucidated. Breastfeeding and local trauma can be present. Other hypotheses include the use of oral contraceptives, hyperprolactinemia, smoking, infection, autoimmune diseases and alpha 1 antitrypsin deficiency. Objectives: To analyze data from patients in Hospital Federal da Lagoa diagnosed with IGM, and compare the results with the literature. METHOD: We conducted a retrospective analysis of the medical charts in Hospital da Lagoa, Rio de Janeiro, between 2013 and 2017, and identified 15 women, aged between 21 and 76 years, with IGM. RESULT: Of the 15 patients, 14 (93.3\%) were tested for mastitis caused by common germs and Koch's bacillus. Thoracic x-ray, PPD and culture were performed, and one patient tested positive for BK. Of the 14 patients with negative result for secondary infection, one was pregnant, one had HIV, and the other 12 (85.7\%) did not present any specificities. At physical examination, 10 women (66.6\%) had ulcer, 9 (60\%) had a palpable nodule. Eleven patients (73.3\%) had undergone an ultrasound, 5 (45.4\%) with BIRADS 4; 4 (36.3\%) with BIRADS 3; one patient (9\%), BIRADS 2, and two (18.1\%), BIRADS 1. In the mammography of the first appointment, 7 (46.6\%) had records int he medical chart. Three (42.8\%) with BIRADS zero; 2 (28.5\%), BIRADS 3; one (14.3\%), BIRADS 4, and another one (14.3\%), BIRADS. Six patients were submitted to some surgical treatment (40\%); regarding medicine treatment, 10 patients had records. One (10\%) received RIP scheme; 4 (40\%) received antibiotic therapy; two (20\%), a combination of antibiotics and corticoids (oral); one (10\%), a combination of methotrexate with topic and oral corticoid; two (20\%, topic corticoid. Conclusion: By comparing the medical charts' review with the literature, the results regarding clinical presentation, diagnosis and treatment are similar. The histopathological study is gold standard for diagnostic confirmation, and the medicinal conduct with regular follow-up is the best treatment. 